\title{
Resonant Orbits in Triaxial Galaxies
}

\author{
David Merritt and Monica Valluri \\ Department of Physics and Astronomy, Rutgers University, New Brunswick, NJ 08855
}

\begin{abstract}
Box orbits in triaxial potentials are generically thin, that is, they lie close in phase space to a resonant orbit satisfying a relation of the form $l \omega_{1}+m \omega_{2}+n \omega_{3}=0$ between the three fundamental frequencies. Boxlets are special cases of resonant orbits in which one of the integers $(l, m, n)$ is zero. Resonant orbits are confined for all time to a membrane in configuration space; they play roughly the same role, in three dimensions, that periodic orbits play in two, generating families of regular orbits when stable and stochastic orbits when unstable. Stable resonant orbits avoid the center of the potential; orbits that are thick enough to pass near the destabilizing center are typically stochastic. Resonances in triaxial potentials are most important at energies far outside the region of gravitational influence of a central black hole. Near the black hole, the motion is essentially regular, although resonant orbits exist in this region as well, including at least one family whose elongation is parallel to the long axes of the triaxial figure.
\end{abstract}

\section{Introduction}

Motion in three-dimensional systems can differ in a number of qualitative ways from motion in one or two dimensions. One example is complex instability (Broucke 1969; Pfenniger 1995), in which perturbations of a periodic orbit diverge exponentially while rotating with a characteristic frequency. Another example is Arnold diffusion (Arnold 1964), the non-isolation of chaotic regions in phase space.

The focus of the present study is on a different property of three-dimensional motion. Associated with each degree of freedom of regular (quasi-periodic) motion is a frequency $\omega_{i}$, the rate of change of the corresponding angle variable. The character of the motion depends critically on whether the $\omega_{i}$ 's are independent, or whether they satisfy one or more nontrivial linear relations of the form

$$
\sum_{i=1}^{N} m_{i} \omega_{i}=0
$$


with $N$ the number of degrees of freedom (DOF) and $m_{i}$ integers, not all of which are zero. Generally there exists no relation like equation (11); the frequencies are incommensurable, and the trajectory fills its torus uniformly and densely in a time-averaged sense. When one or more resonance relations are satisfied, however, the trajectory is restricted to a phase space region of lower dimensionality than $N$.

The importance of resonances for motion in two-dimensional systems is well known: resonant tori - periodic, or closed, orbits - are regions where the independent motions are coupled together, leading to a breakdown in perturbation expansions. The character of the motion near a resonance is described by a number of theorems, including the Poincaré-Birkhoff and Floquet theorems. When stable, resonant tori generate new families of regular orbits whose shape mimics that of the parent periodic orbit. Unstable resonant tori are typically associated with a breakdown of integrability and with chaos. In this sense, periodic orbits provide the phase space of a 2 DOF system with its structure.

In three dimensions, a single resonance relation like equation (11) does not imply that an orbit will be closed; rather, it restricts the orbit to a space of dimension two. An orbit satisying one such relation is therefore "thin," confined for all time to a (possibly self-intersecting) membrane. In order for an orbit in a 3 DOF system to be closed, it must satisfy two such independent relations; only then is the motion confined to a one-dimensional curve. One expects that orbits satisfying two resonance relations will be rare compared to orbits satisfying just one, and hence that thin orbits - rather than periodic orbits - are the objects of fundamental importance in structuring the phase space of 3 DOF systems. We present evidence in support of this hypothesis below.

The emphasis in the present paper is on three-dimensional resonances, i.e., resonances for which each of the integers $m_{i}$ in equation (1) is nonzero. Thin orbits generated from a two-dimensional resonance have been widely studied; examples are the "thin tubes" discussed by Bishop, de Zeeuw and collaborators (Bishop 1987; de Zeeuw \& Hunter 1990; Evans, de Zeeuw \& Lynden-Bell 1990; de Zeeuw, Evans \& Schwarzschild 1996). Thin tube orbits exist even in fully integrable potentials; they are linked to the $1: 1$ resonant orbits in the principal planes of Stäckel models (de Zeeuw 1985). By contrast, most of the resonant orbits discussed here can not be associated with any planar orbit; their existence is a consequence of a coupling between all three degrees of freedom and they have no analog in two-dimensional or in Stäckel potentials.

The importance of resonant orbits in 3 DOF systems was suggested by a number of recent studies (Carpintero \& Aguilar 1998; Papaphilippou \& Laskar 1998; Valluri \& Merritt 1998; Wachlin \& Ferraz-Mello 1998) that applied torus-construction machinery to motion in triaxial potentials. These studies noted that the phase space of box orbits, i.e. orbits 
with a stationary point, is densely structured by resonances, especially in models with central mass concentrations or nuclear "black holes." Valluri \& Merritt (1998) noted that a resonance relation like equation (1) implies a reduction in the dimensionality of an orbit and presented an illustration of a thin box orbit.

Here we study the properties of such orbits in more detail. Section 2 discusses the reduction in dimensionality that follows from the existence of a resonance between the three degrees of freedom. Section 3 presents the properties of thin box orbits in a family of triaxial models with central density cusps and nuclear black holes; the emphasis is on the conditions under which such orbits are stable and can give rise to families of quasi-periodic orbits with finite thickness. Section 4 extends this discussion to the central regions of a triaxial galaxy containing a black hole, where the potential is nearly Keplerian. Some implications for galactic structure and evolution are presented in Section 5.

\section{Resonances}

In this section we discuss the effect of a resonance relation like equation (目) on the motion of a regular orbit, and the differences between resonances in 2 and 3 DOF systems.

For a two-dimensional regular orbit with fundamental frequencies $\omega_{1}$ and $\omega_{2}$, the angle variables are

$$
\theta_{1}=\omega_{1} t, \quad \theta_{2}=\omega_{2} t
$$

which define the surface of a torus. The constants indicating phase on the torus have been set to zero without loss of generality. Because of the quasi-periodicity of the orbit, its torus can be mapped onto a square in the $\left(\theta_{1}, \theta_{2}\right)$-plane, with each side ranging from 0 to $2 \pi$ (Figure 1); the top and bottom of the square are identified with each other, as are the left and right sides. In the general case, the frequencies $\omega_{1}$ and $\omega_{2}$ are incommensurate and the trajectory densely covers the entire $\left(\theta_{1}, \theta_{2}\right)$-plane after an infinite time. However if the ratio $\omega_{1} / \omega_{2}=\left|m_{2} / m_{1}\right|$ is a rational number, i.e. if $m_{1}$ and $m_{2}$ are integers, the orbit closes on itself after $\left|m_{2}\right|$ revolutions in $\theta_{1}$ and $\left|m_{1}\right|$ revolutions in $\theta_{2}$ and fills only a one-dimensional subset of its torus (e.g. Arnold 1973, p. 164). Its dimensionality in configuration space is also one. Such an orbit has a single fundamental frequency $\omega_{0}=\omega_{1} / m_{2}=\omega_{2} / m_{1}=2 \pi / T$, with $T$ the orbital period; after an elapsed time $T$, the trajectory returns to its starting point in phase space.

The effect of resonances on the motion in two-dimensional, non-integrable potentials is well understood (Arnold 1989; Lichtenberg \& Lieberman 1992). Resonant orbits, when stable to perturbations, are associated with families of regular orbits, and when unstable 
generate regions of stochasticity. Examples of resonant orbit families in two-dimensional galactic potentials are the boxlets (Miralda-Escudé \& Schwarzschild 1989) which exist in the principal planes of triaxial models. Two-dimensional resonances in the meridional plane of axisymmetric systems (Evans 1993) and in planar barred potentials (Contopoulos \& Grosbøl 1989) have also been treated in detail.

In the case of a three-dimensional regular orbit, the angle variables are

$$
\theta_{1}=\omega_{1} t, \quad \theta_{2}=\omega_{2} t, \quad \theta_{3}=\omega_{3} t
$$

The orbit may now be mapped into a cube whose axes are identified with the $\theta_{i}$ (Figure 2). If the $\omega_{i}$ are incommensurate, this cube will be densely filled after a long time. However if a single condition of the form

$$
m_{1} \omega_{1}+m_{2} \omega_{2}+m_{3} \omega_{3}=0
$$

is satisfied, with the $m_{i}$ integers (not all of which are zero), the motion is restricted for all time to a two-dimensional subset of its torus (Born 1960, p. 91; Goldstein 1980, p. 470). An example is illustrated in Figure 2, with $\left(m_{1}, m_{2}, m_{3}\right)=(2,1,-2)$. Such an orbit is not closed; instead, as suggested by Figure 2, it is thin, restricted to a sheet or membrane in configuration space, which it fills densely after infinite time.

Just as in the two-dimensional case, the condition (4) may be used to reduce the number of independent frequencies by one. Defining the two "base" frequencies $\mathbb{Q} \omega_{0}^{(1)}, \omega_{0}^{(2)}$ as

$$
\omega_{0}^{(1)}=\omega_{3} / m_{1}, \quad \omega_{0}^{(2)}=\omega_{2} / m_{1},
$$

we may write

$$
\begin{aligned}
& \omega_{1}=-m_{3} \omega_{0}^{(1)}-m_{2} \omega_{0}^{(2)}, \\
& \omega_{2}=m_{1} \omega_{0}^{(2)}, \\
& \omega_{3}=m_{1} \omega_{0}^{(1)} .
\end{aligned}
$$

Since the motion is quasi-periodic, i.e.

$$
\mathbf{x}(t)=\sum_{k} \mathbf{X}_{k} \exp i\left(l_{k} \omega_{1}+m_{k} \omega_{2}+n_{k} \omega_{3}\right) t
$$

\footnotetext{
${ }^{1}$ The term "base frequency" is used here in a similar, but more general, sense than in the study of Carpintero \& Aguilar (1998), who considered only 2D resonances.
} 
with $\left(l_{k}, m_{k}, n_{k}\right)$ integers, it will remain quasi-periodic when expressed in terms of the two base frequencies:

$$
\begin{aligned}
& \mathbf{x}(t)=\sum_{i} \mathbf{X}_{k} \exp i\left[\left(-l_{k} m_{3}+n_{k} m_{1}\right) \omega_{0}^{(1)}+\left(-l_{k} m_{2}+m_{k} m_{1}\right) \omega_{0}^{(2)}\right] t \\
& =\sum_{k} \mathbf{X}_{k} \exp i\left(l_{k}{ }^{\prime} \omega_{0}^{(1)}+m_{k}{ }^{\prime} \omega_{0}^{(2)}\right) t, \\
& =\sum_{k} \mathbf{X}_{k} \exp i\left(l_{k}^{\prime} \theta^{(1)}+m_{k}^{\prime} \theta^{(2)}\right), \\
& l_{k}{ }^{\prime}=-l_{k} m_{3}+n_{k} m_{1}, \quad m_{k}{ }^{\prime}=-l_{k} m_{2}+m_{k} m_{1}, \\
& \theta^{(1)}=\omega_{0}^{(1)} t, \quad \theta^{(2)}=\omega_{0}^{(2)} t \text {. }
\end{aligned}
$$

A Fourier transform of the motion will therefore consist of a set of spikes whose positions in frequency space can be expressed as linear combinations of just two frequencies. The choice of base frequencies made here is clearly not unique, a consequence of the fact that the orbit is not closed.

A relation like equation (4) will be called a "resonance" even though it does not imply that any frequency pair can be expressed as a ratio of integers. The integer vector $\left(m_{1}, m_{2}, m_{3}\right)$ is the order of the resonance; the degeneracy of the resonance is defined as the number of independent resonance relations that are satisfied by the $\omega_{i}$. In the case of twofold degeneracy, two independent resonance relations apply:

$$
\begin{gathered}
m_{1} \omega_{1}+m_{2} \omega_{2}+m_{3} \omega_{3}=0, \\
n_{1} \omega_{1}+n_{2} \omega_{2}+n_{3} \omega_{3}=0,
\end{gathered}
$$

and each frequency $\omega_{i}$ may be expressed as a rational fraction of any other:

$$
\frac{\omega_{1}}{\omega_{3}}=\frac{m_{2} n_{3}-m_{3} n_{2}}{m_{1} n_{2}-m_{2} n_{1}}=\frac{l_{1}}{l_{3}}, \quad \frac{\omega_{2}}{\omega_{3}}=\frac{m_{3} n_{1}-m_{1} n_{3}}{m_{1} n_{2}-m_{2} n_{1}}=\frac{l_{2}}{l_{3}}
$$

with $\left(l_{1}, l_{2}, l_{3}\right)$ integers. The motion is therefore periodic with a single base frequency $\omega_{0}=\omega_{1} / l_{1}=\omega_{2} / l_{2}=\omega_{3} / l_{3}$ and the trajectory is closed. In a system with $N$ degrees of freedom, $N-1$ such conditions are required for closure; only in the 2DOF case does a single resonance condition imply closure. ${ }^{2}$

The reduction of the dimensionality of an orbit in the presence of a resonance has been appreciated at least since Einstein's 1917 paper on rules for classical quantization Einstein

2 J. D. Meiss (private communication) makes a distinction between "resonant," or closed, orbits and "commensurable" orbits which satisfy fewer than $N-1$ relations like equation (4). 
1917; Percival 1977; Contopoulos, Magnenat \& Martinet 1982). But the role of resonant orbits in structuring the phase space of generic, three-dimensional systems is still not well understood. Almost all discussions of resonances in the galactic dynamics literature have focussed on restricted cases: on resonances between only two of the three degrees of freedom (e.g. Carpintero \& Aguilar 1998; Wachlin \& Ferraz-Mello 1998); on doubly-degenerate, i.e. closed, orbits (e.g. Pfenniger 1984; Contopoulos 1986); or on special potentials in which the motion is globally resonant, such as the Kepler potential. The KAM theorem leads us to expect that - in 3 DOF as in 2 DOF systems - resonant tori are regions where perturbation expansions break down, leading to a change in the local structure of phase space. We expect thin orbits to play approximately the same role, in three dimensions, that periodic orbits play in two.

\section{Resonant Box Orbits in a Family of Triaxial Models}

We test this hypothesis by exploring the properties of thin orbits in one family of triaxial potentials. The mass density law from which the potential was generated, via Poisson's equation, has the form

$$
\rho(m)=\frac{(3-\gamma) M}{4 \pi a b c} m^{-\gamma}(1+m)^{-(4-\gamma)}, \quad 0 \leq \gamma<3
$$

with $M$ the total mass and $m=\left[(x / a)^{2}+(y / b)^{2}+(z / c)^{2}\right]^{1 / 2}$ the radius-like variable. This mass model is the generalization to triaxial geometry of the spherical family described by Dehnen (1993). The potential and forces in the triaxial geometry may be expressed in terms of one-dimensional integrals (Merritt \& Fridman 1996). Dehnen's law has a power-law central density dependence which approximates the observed luminosity profiles of early-type galaxies and bulges (Crane et al. 1993; Ferrarese et al. 1994; Merritt \& Fridman 1995; Gebhardt et al. 1996).

To this model was added a central point with mass $M_{h}$. Here and below, units are adopted such that $a=G=M=1 ; M_{h}$ is therefore defined as the mass of the central object in units of the total galaxy mass. Following the usual convention, the $x$ - and $z$-axes are identified with the long and short axes of the figure.

The character of the motion in triaxial Dehnen models, at radii well outside the gravitational radius of influence of the black hole (if present), has been discussed by Wachlin \& Ferraz-Mello (1998) and Valluri \& Merritt (1998). As in those studies, orbits were

integrated from various starting positions with zero initial velocity for $\sim 10^{2}$ orbital periods and their motion analyzed using Laskar's $(1988,1990)$ algorithm, a Fourier technique for 
extracting the fundamental frequencies $\omega_{i}$ of a regular orbit with high precision. The additional techniques described in Valluri \& Merritt (1998) were used to find the integers $\left(l_{k}, m_{k}, n_{k}\right)$ associated with each distinct peak $\omega_{k}$ in the frequency spectrum (equation 7 ). For stochastic orbits, Laskar's technique gives an approximation, valid over the integration interval, of the true (continuous) spectrum. Stochastic orbits were identified by integrating each trajectory for two contiguous time intervals and comparing the "fundamental frequencies" computed over each interval. The change in the "fundamental frequency" associated with the largest amplitude term in the spectrum, $\Delta \omega$, was taken as a measure of the rate of stochastic diffusion in phase space (Laskar 1993).

Figure 3 shows initial condition spaces for two triaxial Dehnen models, the first with $\gamma=0.5$ and $M_{h}=0$, the second with $\gamma=0.5$ and $M_{h}=0.003$. Both models have $c / a=0.5$ and $b / a=0.791$. The top frames (reproduced with slight modification from Valluri \& Merritt 1998) show one octant of the equipotential surface, each located slightly within the half-mass radius of the model (or at "shell 8" in the notation of those authors). On this surface, a grid of $\sim 10^{4}$ orbits were begun with zero velocity and integrated for 100 orbital periods. The density of the gray scale is proportional to the logarithm of the stochastic diffusion rate as measured by $\Delta \omega$ over the integration interval. Initial conditions for which the motion was found to be regular are shown in white.

The model of Figure 3a is close to integrable, with a finite central force and a moderate central force gradient. Most of the significant stochasticity in this model is confined to initial conditions that lie between the short and intermediate axes, the " $Y-Z$ instability strip" first described by Goodman \& Schwarzschild (1981).

Elsewhere on the equipotential surface, one sees a complex network of intersecting resonance zones, some regular (white) and some stochastic (dark). The starting points of the thin orbits lie along the centers of these zones. Several of the most important resonance zones in Figure $3 \mathrm{a}$ are labelled by their defining integers. Three of these - the $(2,0,-1)$ $(x-z$ banana) resonance, the $(4,-3,0)$ ( $x-y$ pretzel) resonance, and the $(3,0,-2)(x-z$ fish) resonance - are families that connect smoothly to periodic orbits in one of the principal planes, i.e. to "boxlets" (Miralda-Escudé \& Schwarzschild 1989). Others - e.g. the $(3,-1,-1),(2,1,-2)$ and $(4,-2,-1)$ resonances - are not related to any planar periodic orbit; these resonances are characterized by nonzero values for each of the integers $m_{i}$.

Examples of thin orbits from each of these six families are shown in Figures 4 and 5. Figure 4 plots intersections of the orbits with the three principal planes; because these orbits are thin, their intersection with any plane defines a curve or set of curves, rather than a finite area as in the case of a volume-filling orbit. None of the orbits passes precisely through the center although all of them come quite close. Figure 5 presents views of 
the surfaces defined by the orbits. These plots were generated using Laskar's algorithm to extract the frequency spectra, equation (17), followed by equation (8) which yields the Cartesian coordinates in terms of the two reduced angle variables $\left(\theta^{(1)}, \theta^{(2)}\right)$. The resulting (numerical) functions $\mathbf{x}\left(\theta^{(1)}, \theta^{(2)}\right)$ define a surface that was plotted via the Mathematica routine "ParametricPlot3D."

When projected against the principal planes, the thinness of these orbits is not readily apparent and it is likely that thin box orbits were seen but not identified as such in many earlier studies. A possible example is shown in Figure 6 of Levison \& Richstone (1987).

While there are initial conditions in Figure 3a that generate closed orbits - orbits restricted to a single curve in configuration space - the majority of regular orbits are identifiable only with a singly-degenerate resonance zone. Further evidence for this interpretation is provided by Figure 6, which shows the frequency spectra of two orbits, computed by Fourier analysis of the $z$-component of the motion. The first orbit is from the regular region that lies at the intersection of the $(2,1,-2)$ and $(4,-2,-1)$ resonance zones in Figure 3a. The intersection of these zones defines a regular region of degeneracy two, associated with the closed, $5: 6: 8$ orbit at its center. The second orbit is from the $(2,1,-2)$ resonance zone; this orbit is not obviously identified with any closed orbit.

Many of the lines in the spectrum of the first orbit, Figure 6a, lie precisely at integer multiples of a single base frequency, $\omega_{k}=n_{k} \omega_{0}^{(1)}$, with $\omega_{0}^{(1)}=0.05997853$. This frequency is close to the (single) frequency of the $5: 6: 8$ periodic orbit whose starting point lies nearby on the equipotential surface. In addition, the spectrum of Figure 6a contains pairs of lines that are offset symmetrically from the primary lines, at frequencies of $\omega_{k} \pm \omega_{0}^{(2)}$ and $\omega_{k} \pm \omega_{0}^{(3)}$, where $\omega_{0}^{(2)}=0.012007$ and $\omega_{0}^{(3)}=0.016179$. These two additional frequencies may be interpreted as resulting from the slow libration, in two independent directions, of the orbit around the parent closed orbit. (Binney \& Spergel (1982) motivate this interpretation in the context of a two-dimensional orbit.) The spectrum of the orbit in Figure $6 \mathrm{a}$ is thus clearly recognizable as that of a perturbed, closed orbit.

By contrast, the spectrum of the second orbit (Figure 6b) contains lines at integer multiples of two base frequencies. These may be defined as $\omega_{0}^{(1)}=\omega_{x}=0.29655554$, the frequency associated with the strongest line in the $x$-spectrum, and $\omega_{0}^{(2)}=\omega_{z}=0.48353497$, the primary frequency of the $z$-motion. The strongest line in the $y$-spectrum lies at $\omega_{y}=-2 \omega_{x}+2 \omega_{y}=-2 \omega_{0}^{(1)}+2 \omega_{0}^{(2)}$, consistent with the location of this orbit within the $(2,1,-2)$ resonance zone. If the orbit whose spectrum is shown in Figure $6 \mathrm{~b}$ were precisely thin, all of its lines would be representable in terms of the two base frequencies, as discussed above. However one observes a third frequency in the form of multiplets at frequencies $l_{k} \omega_{0}^{(1)}+m_{k} \omega_{0}^{(2)} \pm \omega_{0}^{(3)}$, with $\omega_{0}^{(3)}=0.010803$. The "splitting" frequency $\omega_{0}^{(3)}$ is the same 
(within numerical precision) for all of the multiplets of Figure $6 \mathrm{~b}$ and in all three $(x, y, z)$ of the spectra. Thus, this orbit is clearly identifiable as a perturbed thin orbit rather than as a perturbed closed orbit.

Both spectra show additional, higher-order multiplets at low amplitude.

Examination of the spectra of a larger set of orbits suggests that regular orbits whose starting points lie within a (singly-degenerate) resonant zone always have spectra like that of Figure $6 \mathrm{~b}$, i.e. with two base frequencies and a single splitting frequency, rather than like that of Figure 6a, with a single base frequency and two splitting frequencies. In this sense it is reasonable to state that the majority of regular orbits at this energy are associated with thin orbits and not with closed orbits.

A striking feature of Figure 3a is the large number of distinct, and fairly narrow, resonance zones. The reason for the narrowness of the zones is suggested by Figure 3c, which shows the distance of closest approach to the potential center of a set of orbits whose initial conditions lie along the heavy curve in Figure 3a. As one passes through a stable resonance zone, the orbital pericenter distance reaches a maximum on the resonance, where the orbit has zero thickness. Initial conditions that lie to either side of the resonance produce orbits with a finite thickness; as this thickness increases, the pericenter distance falls, and eventually the orbit becomes thick enough to pass through the center of the potential. Orbits with pericenter distances close to zero are generally stochastic, as shown in Figure $3 \mathrm{e}-\mathrm{a}$ likely consequence of the steepness of the force gradient near the center, which causes the trajectory to become sensitive to small perturbations. The precise distance from the center at which stochasticity sets in is different for each resonant family but is typically of order $\sim 0.005$ at this energy in this potential. (The linear scale of the orbits is of order 1 at this energy). The narrowness of the resonance zones is therefore a consequence of the fact that only a slight offset of an orbit's starting point from resonance is sufficient to force it into the destabilizing center.

The space between the primary resonance zones in Figure 3a appears to be criss-crossed by a large number of narrower zones. Some of these additional zones are identified in Figure $3 \mathrm{c}$ with their integer vectors $\left(m_{1}, m_{2}, m_{3}\right)$. A further illustration of the dense packing of resonance zones is given in Figure 7 , which shows the transition between the $(2,1,-2)$ and $(4,-2,-1)$ resonant orbits along the heavy line of initial conditions shown in Figure 3a. As one moves between these two primary zones, one passes over the two subsidiary zones - with orders $(-5,4,0)$ and $(1,3,-3)$ - which can also be clearly seen in the pericenter plot, Figure 3c. But resonances of even higher order are apparent in Figure 7. The only orbits in that figure that appear to be genuinely volume-filling - numbers 11 and 16 - are slightly stochastic, although weakly enough that their stochasticity does not allow them 
to visit the full volume defined by the equipotential surface over an integration interval of $\sim 10^{2}$ oscillations. (The weak stochasticity is a consequence of the low degree of central concentration of this model, $\gamma=0.5$, and the absence of a central "black hole.") Thus, essentially every regular orbit in this set appears to be associated with a resonance.

The denseness of the resonance zones is not surprising. In an integrable potential, where the fundamental frequencies vary smoothly with initial conditions, it is well known that resonant tori are dense in the phase space, just as rational numbers are dense in the space of real numbers. Even a slight perturbation of the potential away from exact integrability would be expected to radically change the motion in the neighborhood of each of these resonances, in the same way that the motion in perturbed 2D systems is strongly affected by the existence and stability of closed orbits.

Some of the resonance zones in Figure $3 \mathrm{a}$ are present also in Figure 3b, which shows an equipotential surface in the model with an added central point mass, $M_{h}=0.003$. However the higher-order resonance zones (e.g. $(6,-6,1),(-5,4,0))$ have disappeared; the motion in the corresponding regions is now chaotic. A second difference is that - within a given resonance zone - chaos sets in well before an orbit is thick enough to sample the center (Figure 3d, f). Evidently, an added central mass point can induce stochasticity even in orbits that do not pass particularly close to the center. Figure 8 shows the approximate pericenter distance at which integrability is destroyed for three of the resonant orbit families in Figure 3b, as a function of the central mass $M_{h}$; in each of these potentials, the amplitude of the long-axis orbit is about 2 . When $M_{h}$ exceeds $\sim 1 \%$ the mass of the galaxy - typical of the black holes in a number of early-type galaxies (Ford et al. 1998) - a pericenter distance of $\sim 5 \%$ of the orbital amplitude is sufficient to induce stochasticity for each of these families. The highest-order resonant family in Figure 8 , the $(4,-2,-1)$ family, has been rendered completely chaotic for $M_{h} \gtrsim 0.01$, and the other families disappear for $M_{h} \gtrsim 0.03$.

Valluri \& Merritt (1998) reported a transition to global stochasticity in the phase space of box orbits when the mass of a central point exceeded $\sim 1-3 \%$ the galaxy mass. Figure 8 suggests a simple explanation for this transition: when the central mass is sufficiently great, even resonant orbits are unable to avoid the center by a wide enough margin to remain stable.

It was argued above that the majority of regular orbits in these potentials are properly associated with thin orbits rather than with closed orbits. Further evidence in support of this claim is presented in Figure 9, which shows the variation of the splitting frequency $\omega_{0}^{(3)}$ defined above with initial conditions as one moves across the $(2,1,-2)$ resonance zone in the potential with $\gamma=0.5$ and $M_{h}=0.0003$. The variation in $\omega_{0}^{(3)}$ is generally smooth, peaking on the resonance and falling off to either side. This smooth variation suggests a 
continuous dependence of orbital properties on initial coordinates near the resonance. One also sees some discontinuities; inspection of the individual orbits reveals the existence of additional resonances, i.e. closed orbits, at these points. These closed orbits must in some sense be dense in phase space. However the generally smooth variation of $\omega_{0}^{(3)}$ with initial conditions suggests that the majority of orbits in a singly-degenerate resonance zone can be usefully associated with the resonance.

\section{Orbits near the Central Black Hole}

The character of the orbits, as well as the relative importance of thin orbits, might be expected to change as one approaches the center of a triaxial potential containing a nuclear black hole. Pfenniger \& de Zeeuw (1989) and Sridhar \& Touma (1998) noted that the motion in the neighborhood of the black hole, i.e. at radii such that the mass of the black hole is comparable to or greater than the enclosed mass in stars, should be approximately integrable, and these authors presented the results of numerical integrations in two-dimensional harmonic-oscillator potentials with added central point masses.

We begin by describing how the population of box orbits changes as one moves from the half-mass radius into the region where the forces are dominated by the black hole. We chose a Dehnen model with $\gamma=0.5$ and $M_{h}=0.003$; the latter value is typical of the black hole mass ratio in early-type galaxies (Ford et al. 1998), and the weak cusp specified by $\gamma=0.5$ is characteristic of bright elliptical galaxies (Gebhardt et al. 1996), in which the evolution timescales due to chaotic mixing are long enough that a triaxial shape might maintain itself for roughly a Hubble time (Merritt \& Quinlan 1998; Valluri \& Merritt 1998). Following Merritt \& Fridman (1996), the mass model without the black hole was divided into 21 ellipsoidal shells of equal mass; thus the outer edge of shell 1 contains $1 / 21$ of the total mass and shell 21 lies at infinity. The energy corresponding to each shell was defined as the value of the gravitational potential - now including the contribution from the central black hole - on the $x$-axis at the outer edge of the shell. The ratio of $M_{h}$ to the stellar mass enclosed by shell I, $M_{h} / M_{I}$, is then $21 \times 0.003 / I=0.063 / I$.

At shell $8\left(M_{h} / M_{I}=0.0079\right)$, the diffusion rate map is given by Figure $3 \mathrm{~b}$. As the energy is reduced, the fraction of the starting points on the equipotential surface that generate regular motion drops; by shell $2\left(M_{h} / M_{I}=0.031\right)$, the motion is almost entirely chaotic, containing only small regular regions associated with the $(2,0,-1),(3,0,-2)$ and $(3,-1,-1)$ resonances. At shell $1\left(M_{h} / M_{I}=0.063\right)$ the motion of box orbits is essentially fully stochastic. 
This transition to global stochasticity in the phase space of box orbits as the energy is reduced is similar to the change observed by Valluri \& Merritt (1998), at a fixed energy (shell 8), as $M_{h}$ was increased. Those authors found that the motion of box orbits became almost completely stochastic when the mass of the black hole increased past $\sim 0.01$ times the total mass of the model, or equivalently, $\sim 0.02$ times the enclosed mass. We speculate that a transition to global stochasticity in the phase space of box orbits generically occurs at radii where the black hole contains of order $\sim 1-3 \%$ of the enclosed mass in triaxial potentials.

Inside of this radius, a zone of chaos was found to extend inwards, roughly to the radius at which the gravitational force from the black hole begins to dominate the force from the stars. To explore this central region, we defined a new set of shells, denoted by the index $J$, which divide the previously-innermost shell $(I=1)$ again into 21 equal-mass shells; thus shell $J=1$ encloses $(J / 21) \times(1 / 21)=0.0023$ of the total stellar mass, etc. The ratio of the black hole mass to the enclosed stellar mass within this region is therefore $M_{h} / M_{J} \approx 1.323 / J$.

Figure 10 illustrates the behavior of the box orbits as one moves from the inner edge of the zone of chaos, at shell $J=7\left(M_{h} / M_{J} \approx 0.189\right)$, into shell $J=4\left(M_{h} / M_{J} \approx 0.331\right)$. In addition to plots of the diffusion rate, as in Figure 3, we show the "frequency maps" defined by Papaphilippou \& Laskar (1998). The first regular orbits to appear inside of the zone of chaos are associated with the $(2,0,-1)$ resonance, the $x-z$ banana orbit. Unlike the banana orbit at higher energies, which lie close to the long $(x)$ axis, this banana orbit has its stationary point near the short $(z)$ axis of the model. As one moves inward, a regular region appears around the short axis, and grows to include most of the equipotential surface, with the exception of a strip connecting the $x$ and $y$ axes. A number of stable and unstable resonances can be seen at each shell but most of these are important only over a very narrow range of energies; the only stable resonance that persists over a signficant radial range is the $(1,-2,1)$ resonance. The rapid variation in the resonance zones as the energy is reduced corresponds to a shift in the fundamental frequencies toward $\omega_{x} \approx \omega_{y} \approx \omega_{z}$, the expected behavior as one approaches the Keplerian potential of the black hole.

We note that passage through the "zone of chaos" has the effect of reversing the dynamical roles of the long and short axes. At large energies, motion is stable (unstable) in the vicinity of the long (short) axes, respectively, and an instability strip extends from the short to the intermediate axes (Goodman \& Schwarzschild 1981; Figure 3). Inside the zone of chaos, motion near the short axis becomes stable, and the instability strip extends from the intermediate to the long axes (Figure 10). As noted above, the $x-z$ banana orbit also changes its direction of elongation, from the long to the short axis. Sridhar \& Touma 
(1998) noted, in their study of two-dimensional motion in the vicinity of a black hole, that the regular orbits are often elongated in the direction of the shorter of the two axes; our results suggest that the same is true with regard to the minor axis of a triaxial galaxy, at least in the case of the particular family of potentials investigated here.

The two most important families of boxlike orbits near the black hole are illustrated in Figure 11. The first family (Figure 11a) consists of regular orbits not associated with any low-order resonance; similar orbits in two dimensions were called "lenses" by Sridhar \& Touma (1998). Those authors noted that 2D lens orbits could be approximately described as precessing Keplerian ellipses with one focus on the black hole. The orbits found here appear to be straightforward generalizations, to three dimensions, of Sridhar \& Touma's lenses, precessing independently in the $y-z$ and $x-z$ planes. These orbits are essentially volume-filling although close inspection of their cross-sections reveals that many are associated with a high-order resonance.

The second major family consists of orbits associated with the $(1,-2,1)$ thin box mentioned above. The resonant orbits that give rise to this family (Figure 11b) are elongated parallel to the $x-y$ plane; the symmetric objects formed by superposition of four such orbits, reflected about the principal planes, might be useful for self-consistently reconstructing a triaxial figure. The (symmetrized) lens orbits are elongated parallel to the short axis of the triaxial figure and would probably not be very useful for this purpose, as noted by Sridhar \& Touma (1998).

\section{Summary and Discussion}

Our principal conclusions follow.

1. Resonant orbits - orbits confined for all time to a membrane in configuration space play roughly the same role, in three dimensions, that periodic orbits play in two, generating families of regular orbits when stable and stochastic orbits when unstable.

2. Box orbits in realistic triaxial potentials are generically thin. Their thickness is limited by the requirement that they avoid the destabilizing center of the potential; orbits that pass too near the center become stochastic. In triaxial mass models with central "black holes" containing $\sim 0.1 \%-1 \%$ of the total mass, box orbits become stochastic when their distance of closest approach to the center is $\sim 0.05-0.1$ times the half-mass radius of the model.

3. Resonances in triaxial potentials are less important at energies where the 
gravitational potential is dominated by the central black hole. In these central regions, most of the boxlike orbits are regular and associated with a single non-resonant family. However resonant orbits still exist, including at least one family whose primary elongation is perpendicular to the short axis of the galaxy figure.

Resonances in triaxial potentials may be relevant to the following problems of current interest.

1. The rates of many physical processes depend on the efficiency with which stars are supplied to the very center of a galaxy. Examples are tidal disruption (Rees 1992) and accretion (Norman \& Silk 1983) of stars by a black hole, transfer of energy from stars to a binary black hole (Quinlan 1996), interaction of stars with an accretion disk (Ostriker 1983), etc. In a triaxial galaxy, the rates of these processes would depend sensitively on the fraction of stars associated with resonant orbits.

2. It is sometimes argued (e.g. de Zeeuw 1996) that the effectiveness of central density cusps or black holes at inducing changes in the orbital structure of a triaxial galaxy should be lessened by figure rotation, because the Coriolis force in a rotating potential tends to make the box orbits "centrophobic." As shown here, the regular box orbits even in a nonrotating potential are generically centrophobic; hence one might not expect figure rotation to have any great effect on their behavior. In fact a recent study (Valluri 1999) finds that stochasticity becomes more prevalent as the rate of figure rotation is increased, apparently because the Coriolis force tends to broaden orbits that would otherwise be thin, thus driving them into the center.

3. As illustrated in Figure 8, one can compute fairly precisely the distance of closest approach to the potential center at which a regular box orbit becomes stochastic. This distance can be relatively large, of order $10 \%$ the galaxy's half-mass radius, when the black hole contains $\sim 1 \%$ the mass of the galaxy. It follows that the effect of a central mass on the orbital structure of a triaxial galaxy need not be strongly dependent on the spatial scale of the mass distribution. In fact, $N$-body simulations reveal that the response of an initially triaxial galaxy to a central accumulation of mass is essentially independent of whether the mass is distributed (e.g. Katz \& Gunn 1991; Udry 1993; Dubinski 1994) or concentrated in a point (Merritt \& Quinlan 1998).

This work was supported by NSF grant AST 96-17088 and by NASA grant NAG 5-6037. The authors are happy to acknowledge useful discussions with A. Bahri, G. Contopoulos, J. Laskar, R. de la Llave, J. Meiss, J. Moser, R. Nityananda, and Y. Papaphilippou. 


\section{REFERENCES}

Arnold, V. I. 1964, Russian Math. Surveys, 18, 85

Arnold, V. I. 1973, Ordinary Differential Equations (Cambridge: MIT Press)

Arnold, V. I. 1989, Mathematical Methods of Classical Mechanics (New York: Springer)

Binney, J. \& Spergel, D. 1982, ApJ, 252, 308

Bishop, J. 1987, ApJ, 322, 618

Born, M. 1960, The Mechanics of the Atom (New York: Frederick Ungar)

Broucke, R. 1969, Amer. Inst. Aeronautics and Astronautics J. 7, 1003

Carpintero, D. D. \& Aguilar, L. A. 1998, MNRAS, 298, 1

Contopoulos, G. 1986, Astron. Astrophys., 161, 244

Contopoulos, G. 1996, Cel. Mech., 64, 66

Contopoulos, G. \& Grosbøl, P. 1989, Astron. Astrophys. Rev., 1, 261

Contopoulos, G., Magnenat, M. \& Martinet, M. 1982, Physica D6, 126

Crane, P. et al. 1993, AJ, 106, 1371

de Zeeuw, T. 1985, MNRAS, 216, 273

de Zeeuw, T. 1996, in Gravitational Dynamics, Proc. of the 36th Herstmonceux Conference, ed. O. Lahav, E. Terlevich \& R. Terlevich (Cambridge: Cambridge Univ. Press)

de Zeeuw, T. \& Evans, N. W. \& Schwarzschild, M. 1996, MNRAS, 280, 903

Dehnen, W. 1993, MNRAS, 265, 250

de Zeeuw, T. \& Hunter, C. 1990, ApJ, 356, 365

Dubinski, J. 1994, ApJ, 431, 617

Einstein, A. 1917, Verhand. Deut. Phys. Ges. 19, 82

Evans, N. W. 1993, MNRAS, 260, 191

Evans, N. W., de Zeeuw, P. T. \& Lynden-Bell, D. 1990, MNRAS, 244, 111 
Ferrarese, L. et al. 1994, AJ, 108, 1598

Ford, H. C., Tsvetanov, Z. I., Ferrarese, L. \& Jaffe, W. 1998, in IAU Symp. 184, The Central Regions of the Galaxy and Galaxies

Gebhardt, K. et al. 1996, AJ, 112, 105

Goldstein, H. 1980, Classical Mechanics (2nd ed.) (Reading: Addison-Wesley)

Goodman, J. \& Schwarzschild, M. 1981, ApJ, 245, 1087

Katz, N. \& Gunn, J. E. 1991, ApJ, 377, 365

Laskar, J. 1988, A\&A, 198, 341

Laskar, J. 1990, Icarus, 88, 266

Laskar, J. 1993, Physica D, 67, 257

Levison, J. F. \& Richstone, D. O. 1987, ApJ, 314, 476

Lichtenberg, A. J. \& Lieberman, M. A. 1992, Regular and Chaotic Dynamics (Berlin: Springer)

Merritt, D. \& Fridman, T. 1995, A. S. P. Conf. Ser. Vol. 86, Fresh Views of Elliptical Galaxies, ed. A. Buzzoni, A. Renzini \& A. Serrano (Provo: Astronomical Society of the Pacific), 13

Merritt, D. \& Fridman, T. 1996, ApJ, 460, 136

Merritt, D. \& Quinlan, G. 1998, ApJ, 498, 625

Miralda-Escudé, J. \& Schwarzschild, M. 1989, ApJ, 339, 752

Norman, C. A. \& Silk, J. 1983, ApJ, 266, 502

Ostriker, J. P. 1983, ApJ, 273, 99

Papaphilippou, Y. \& Laskar, J. 1998, A\&A, 329, 451

Percival, I. C. 1977, in Advances in Chem. Phys., ed. I. Prigogine \& S. A. Rice, vol. 36, 1

Pfenniger, D. 1984, A\& A, 134, 373

Pfenniger, D. 1995, in Three-Dimensional Systems, Ann. NYAS, vol. 751, ed. H. E. Kandrup, S. T. Gottesman \& J. R. Ipser, 131 
Pfenniger, D. \& de Zeeuw, T. 1989, in Dynamics of Dense Stellar Systems, ed. D. Merritt (Cambridge: Cambridge University Press), 81

Quinlan, G. 1996, New A., 1, 35

Rees, M. 1992, in Physics of Active Galactic Nuclei, eds. W. J. Duschl \& S. J. Wagner (Berlin: Springer), 662

Sridhar, S. \& Touma, J. 1997, MNRAS, 287, L1

Sridhar, S. \& Touma, J. 1998, astro-ph/9811304

Udry, S. 1993, A\& A, 268, 35

Valluri, M. 1999, astroph/9901385

Valluri, M. \& Merritt, D. 1998, ApJ, 506, 686

Wachlin, F. C. \& Ferraz-Mello, S. 1998, MNRAS, 298, 22 
Fig. 1.-

A two-dimensional torus, shown here as a square with identified edges. The plotted trajectory satisfies a 2:1 resonance between the fundamental frequencies, $\omega_{1}-2 \omega_{2}=0$. The trajectory repeats after one rotation in $\theta_{1}$ and two rotations in $\theta_{2}$. The corresponding orbit (e.g. a banana) is closed in configuration space and confined to a one-dimensional curve.

Fig. 2.-

A three-dimensional torus, shown here as a cube with identified sides. The shaded region is covered densely by a resonant trajectory for which $2 \omega_{1}+\omega_{2}-2 \omega_{3}=0$. This trajectory is not closed, but it is restricted by the resonance condition to a two-dimensional subset of the torus. The orbit in configuration space is thin, i.e. confined to a membrane.

Fig. 3.-

Properties of box orbits in triaxial Dehnen models with $\gamma=0.5$. Left panels: $M_{h}=0$; right panels: $M_{h}=0.003$. (a), (b): One octant of the equipotential surface, on which orbits were started with zero velocity. The top, left and right corners correspond to the $z$ (short), $x$ (long) and $y$ (intermediate) axes. The grey scale is proportional to the logarithm of the diffusion rate of orbits in frequency space; initial conditions corresponding to regular orbits are white. The most important resonance zones are labelled with their defining integers $\left(m_{1}, m_{2}, m_{3}\right)$. (c), (d): Pericenter distance $\Delta$ of orbits whose starting points lie along the heavy lines in (a) and (b). The most important stable resonances are again labelled. (e), (f): Degree of stochasticity of the orbits in (c) and (d), as measured by the change $\delta \omega$ in their "fundamental frequencies." $\omega_{0}$ is the frequency of the long-axis orbit. Regular orbits have $\delta \omega / \omega_{0}=0$.

Fig. 4.-

Intersections of six thin box orbits from the potential of Figure 3a with the principal planes. Because the orbits are thin, their intersections with any plane define a curve or set of curves. Each of these orbits is stable and avoids the center, whose position is indicated with a cross.

Fig. 5.-

Plots of the surfaces filled by the thin box orbits whose cross sections are shown in Figure 4, as seen from vantage points on each of the three principal axes.

Fig. 6.- 
Schematic frequency spectra of the $z$-motion of two non-resonant orbits from the potential of Figure 3a. (a) An orbit that lies close to the $5: 6: 8$ closed orbit; (b) an orbit that lies close to a thin orbit from the $(2,1,-2)$ resonance zone. The amplitudes of the frequency spikes have been set to a constant value and only the absolute values of the frequencies are plotted. The "primary" lines of each spectrum are plotted in bold. In (a), these lines lie at integer multiples of $\omega_{0}^{(1)}$, as indicated. In (b), the primary lines lie at $\omega_{k}=l_{k} \omega_{0}^{(1)}+m_{k} \omega_{0}^{(2)}$; each such line is labelled by $l_{k}$ and $m_{k}$. The majority of regular orbits in the potentials investigated here have spectra similar to (b), indicating that they are associated with a thin (singly-degenerate), rather than with a closed (doubly-degenerate), orbit.

Fig. 7.-

$x-y$ cross sections of a set of orbits whose starting points lie equally-spaced on the heavy line of Figure 3a, between the $(2,1,-2)$ and $(4,-2,-1)$ resonance zones. This potential has no central "black hole" and the cusp is weak, $\gamma=0.5$, making the motion nearly integrable. Orbit 7 lies close to the $(-5,4,0)$ resonant orbit, and orbit 13 is close to the $(1,3,-3)$ resonance. Many of the other orbits (e.g. nos. 6, 9, 10) can be assigned to higher-order resonances. Orbits 5, 11 and 16 are weakly stochastic.

Fig. 8.-

Approximate pericenter distance $\Delta_{s}$ at which orbits from three resonant families become stochastic, as a function of the mass $M_{h}$ of a central black hole. $\Delta_{s}$ was found by moving along a line of initial conditions like that of Figure 3b and recording the minimum pericenter distance at which orbits from the resonant family were regular. The amplitude of the long-axis orbit is roughly 2 in each of these model potentials. For $M_{h} \gtrsim 0.01$, the $(4,-2,-1)$ family is fully stochastic; the other two families are stochastic for $M_{h} \gtrsim 0.03$.

Fig. 9.-

Variation of the splitting frequency $\omega_{0}^{(3)}$ with starting point as one moves across the $(2,1,-2)$ resonance zone in the potential with $\gamma=0.5$ and $M_{h}=0.0003$. The position of the resonant orbit is indicated by the thin vertical line. Most orbits with numbers lying between 10 and 80 are regular. Discontinuities occur when a second resonance condition is satisfied, producing a closed orbit.

Fig. 10.-

Properties of boxlike orbits near the central black hole in a triaxial galaxy. The potential is that corresponding to a Dehnen model with $\gamma=0.5$ and $M_{h}=0.003$. Left 
panels show one octant of the equipotential surface with the grey scale indicating the degree of stochasticity. The top, left and right corners correspond to the $z$ (short), $x$ (long) and $y$ (intermediate) axes. Right panels are the frequency maps. The most important resonance zones are labelled with their defining integers $\left(m_{1}, m_{2}, m_{3}\right)$. (a) Shell $J=7$ $\left(M_{h} / M_{J}=0.189\right) ;(\mathrm{b})$ shell $6\left(M_{h} / M_{J}=0.221\right) ;(\mathrm{c})$ shell $5\left(M_{h} / M_{J}=0.265\right) ;(\mathrm{d})$ shell 4 $\left(M_{h} / M_{J}=0.331\right)$.

Fig. 11.-

Non-resonant (a) and resonant (b) orbits near the center of a triaxial galaxy containing a nuclear black hole. The orbit in (b) is associated with the $(1,-2,1)$ resonance. Both orbits are taken from the set whose properties are displayed in Figure 10d. Top panels are projections along the principal axes and bottom panels show intersections with the three principal planes. The non-resonant orbit (a) is volume-filling while the resonant orbit (b) is thin. The position of the black hole is marked with a cross. 


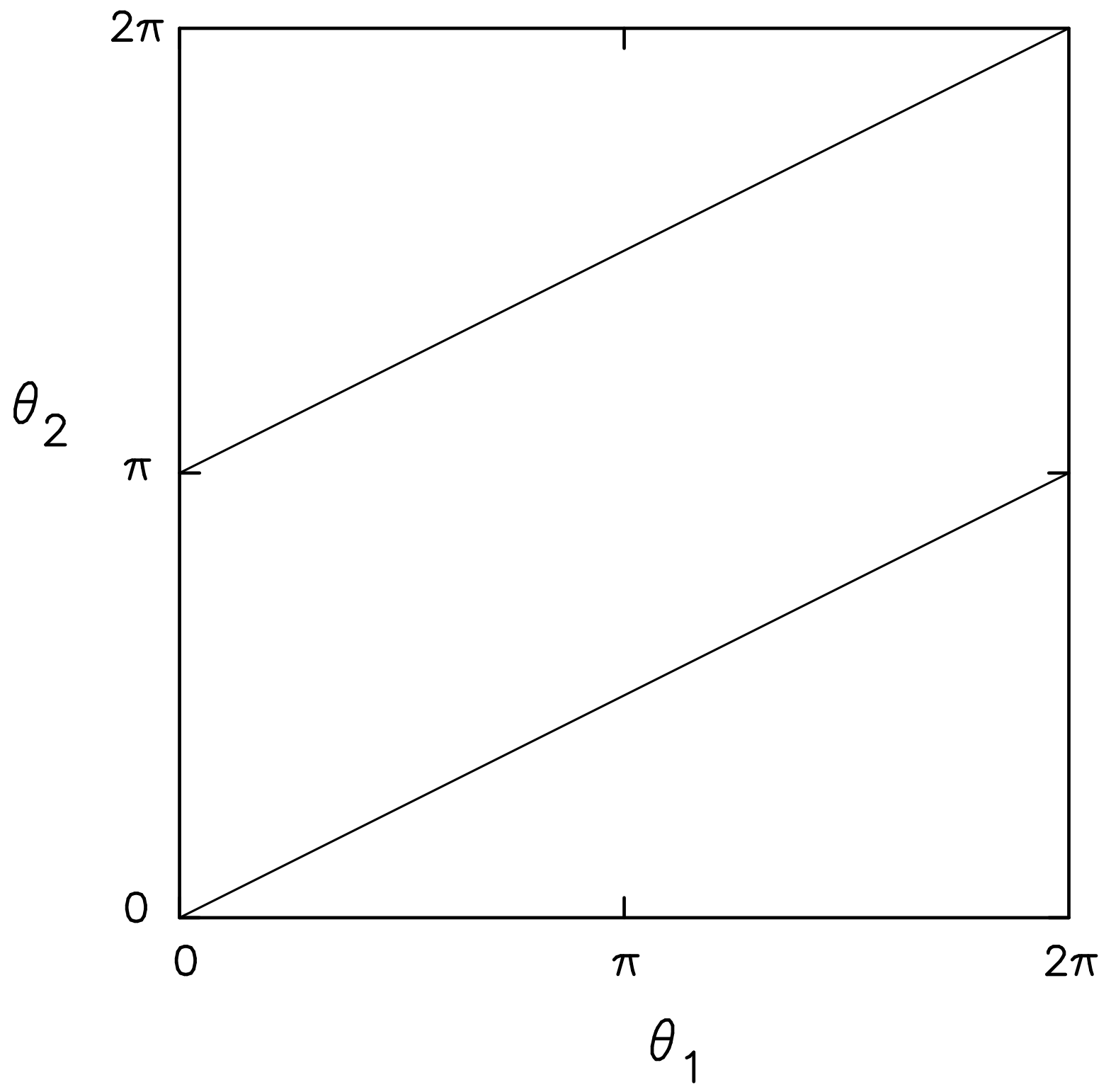

Fig. 1.- 


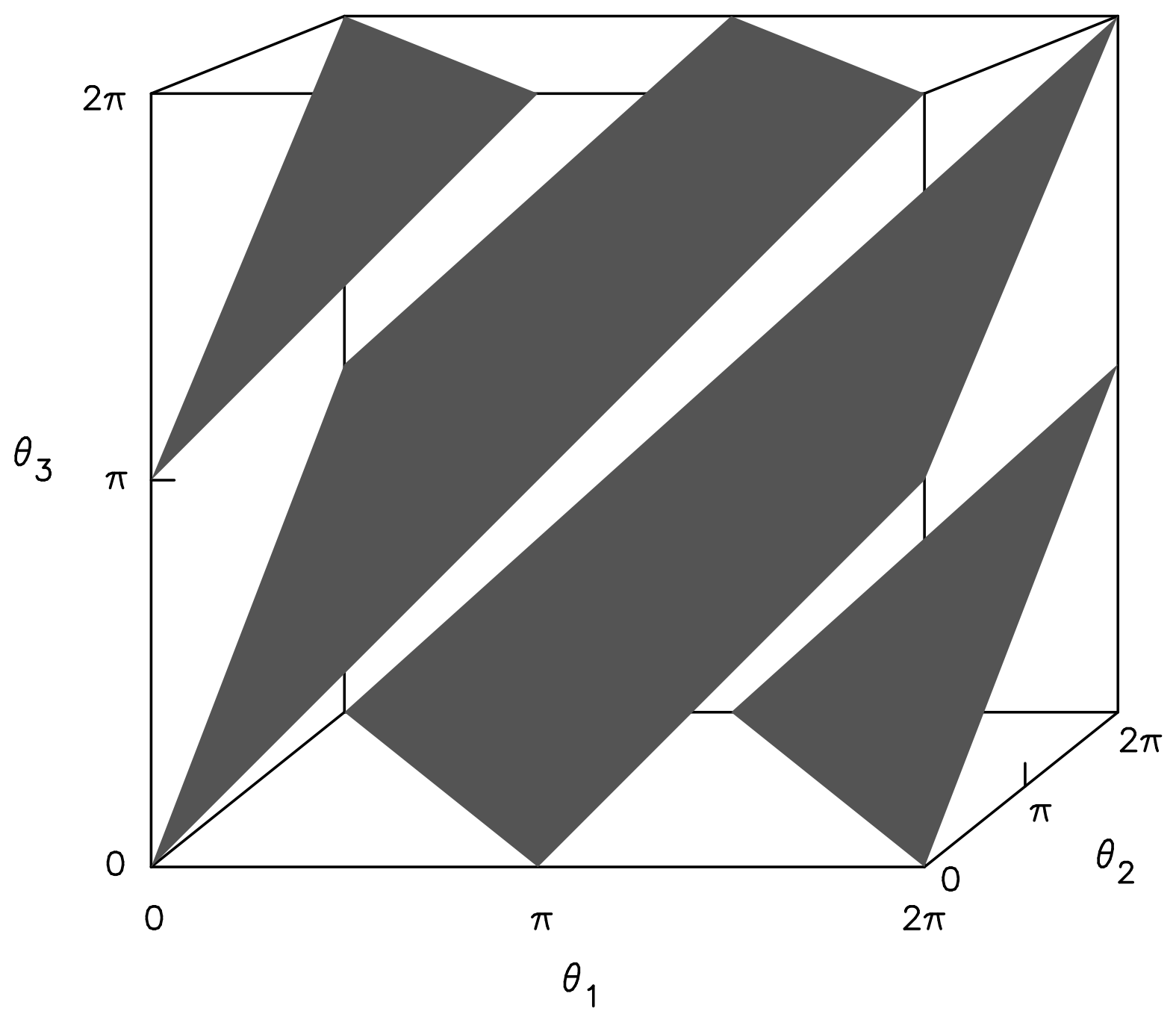

Fig. 2.- 


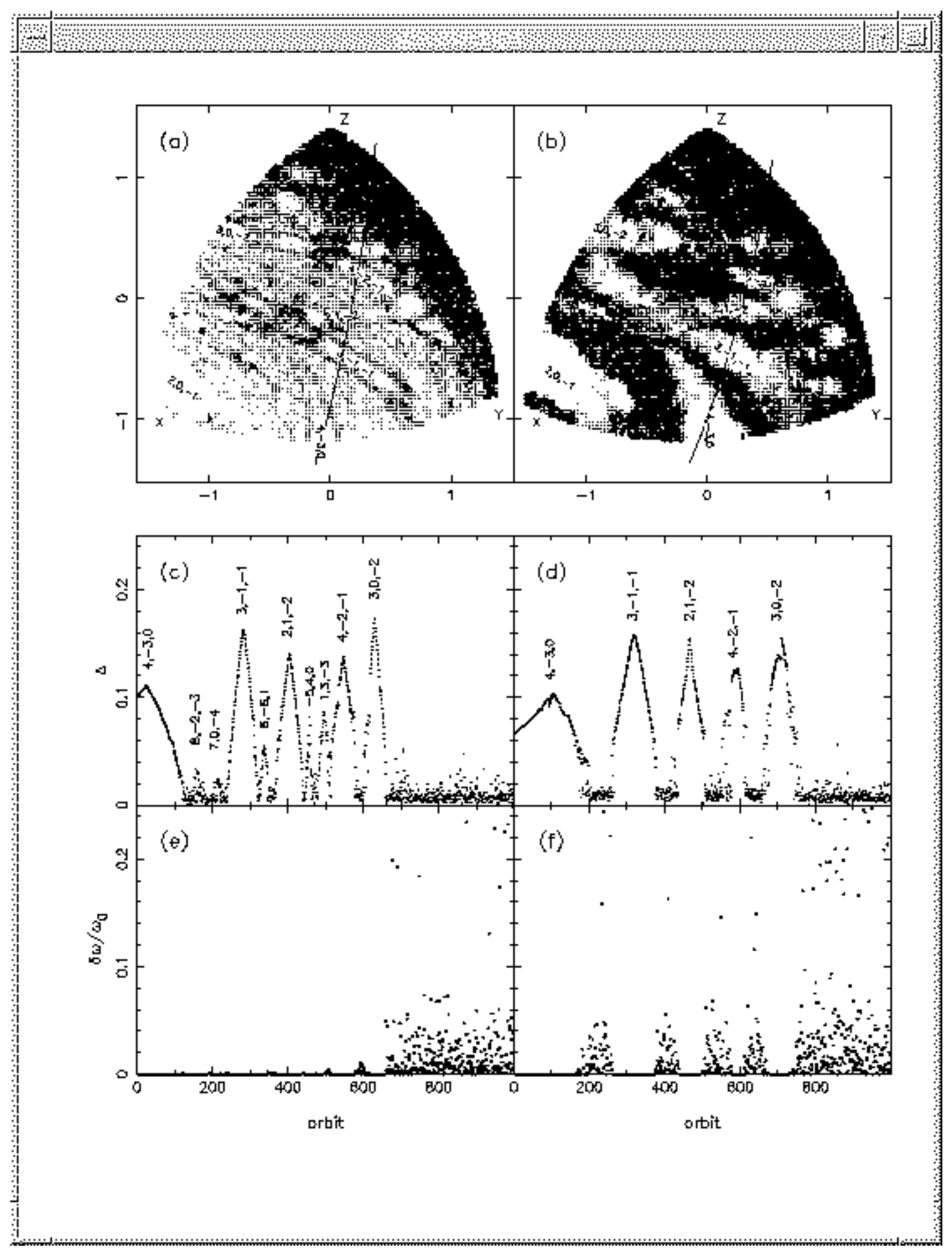

Fig. 3.- 


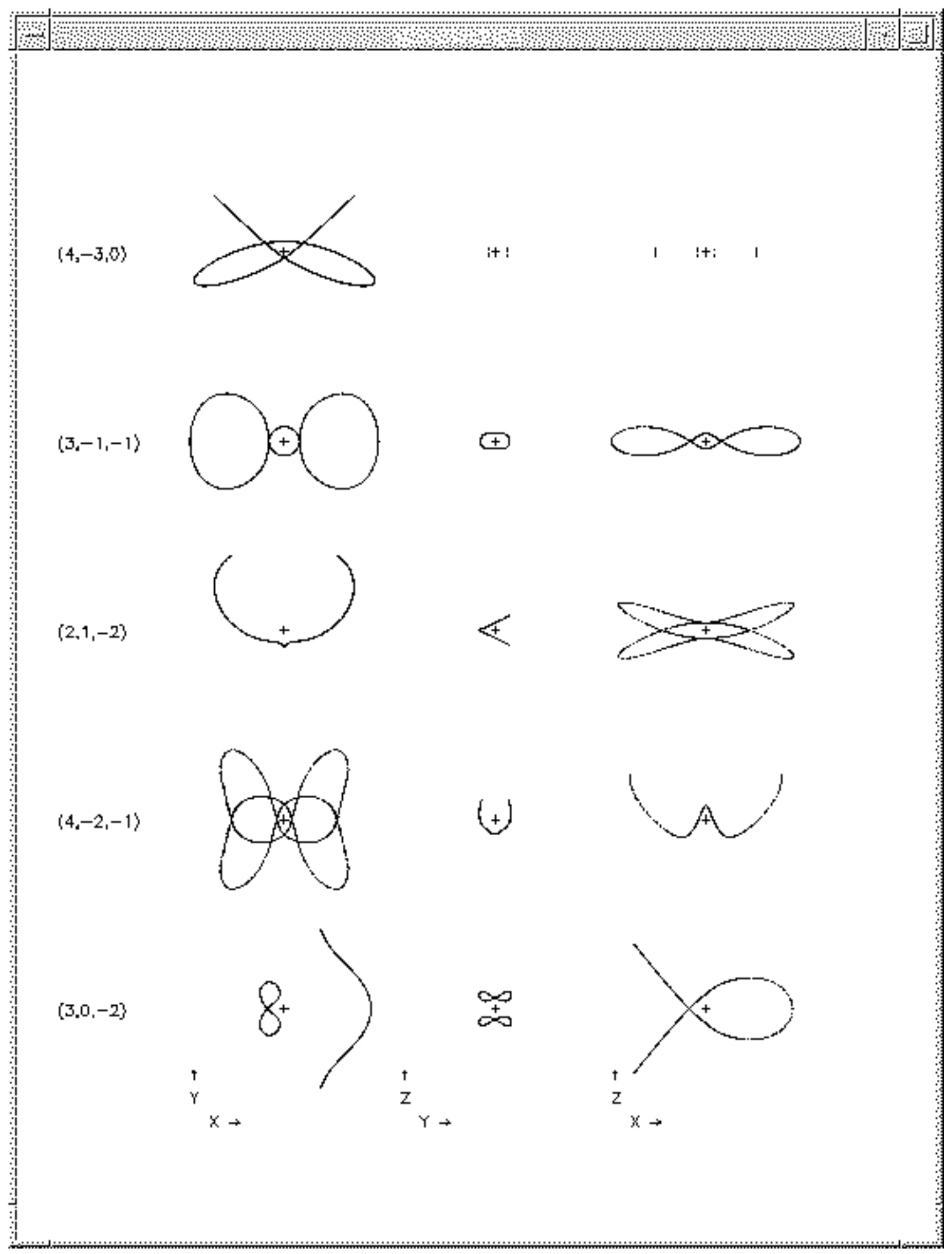

Fig. 4.- 


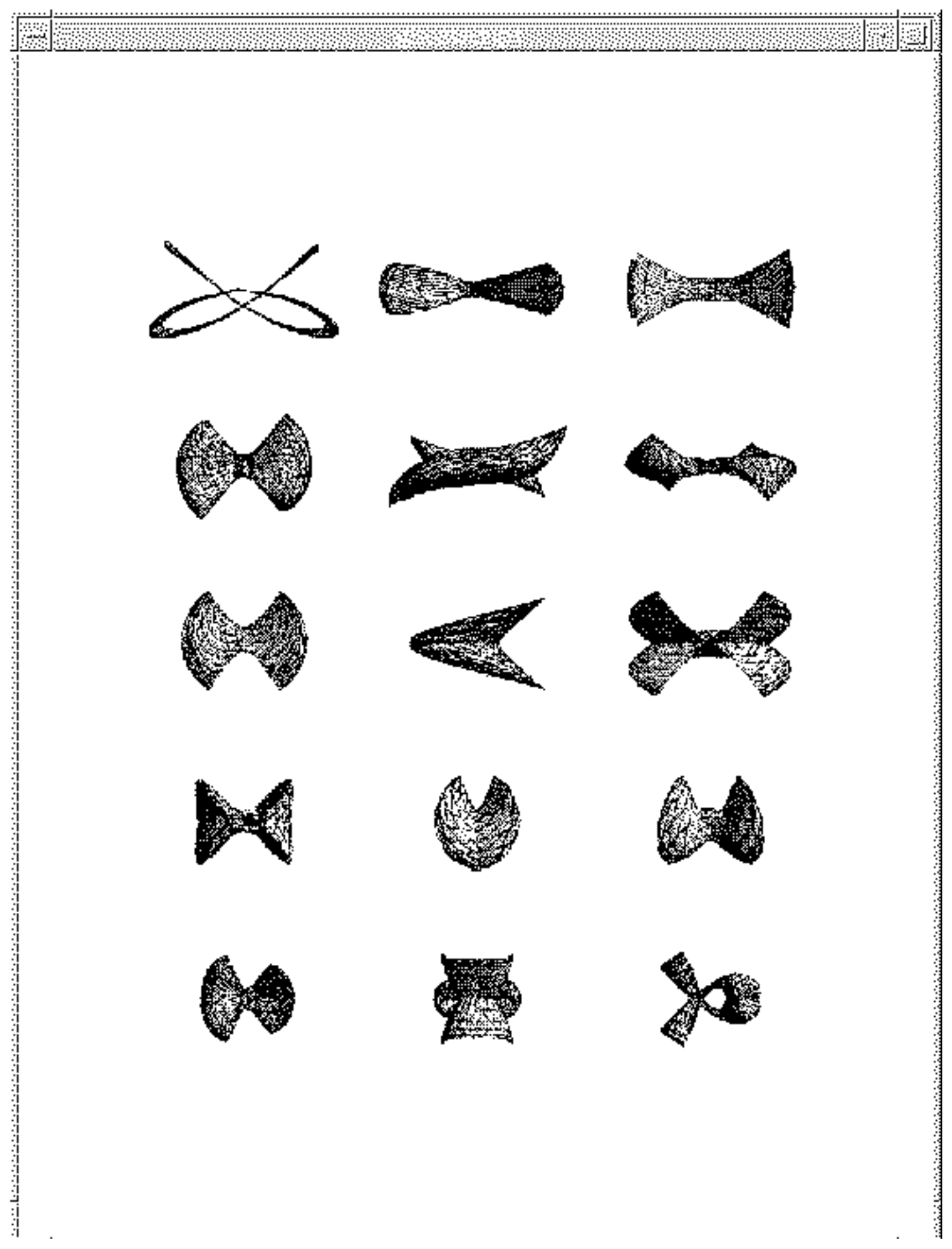

Fig. 5.- 

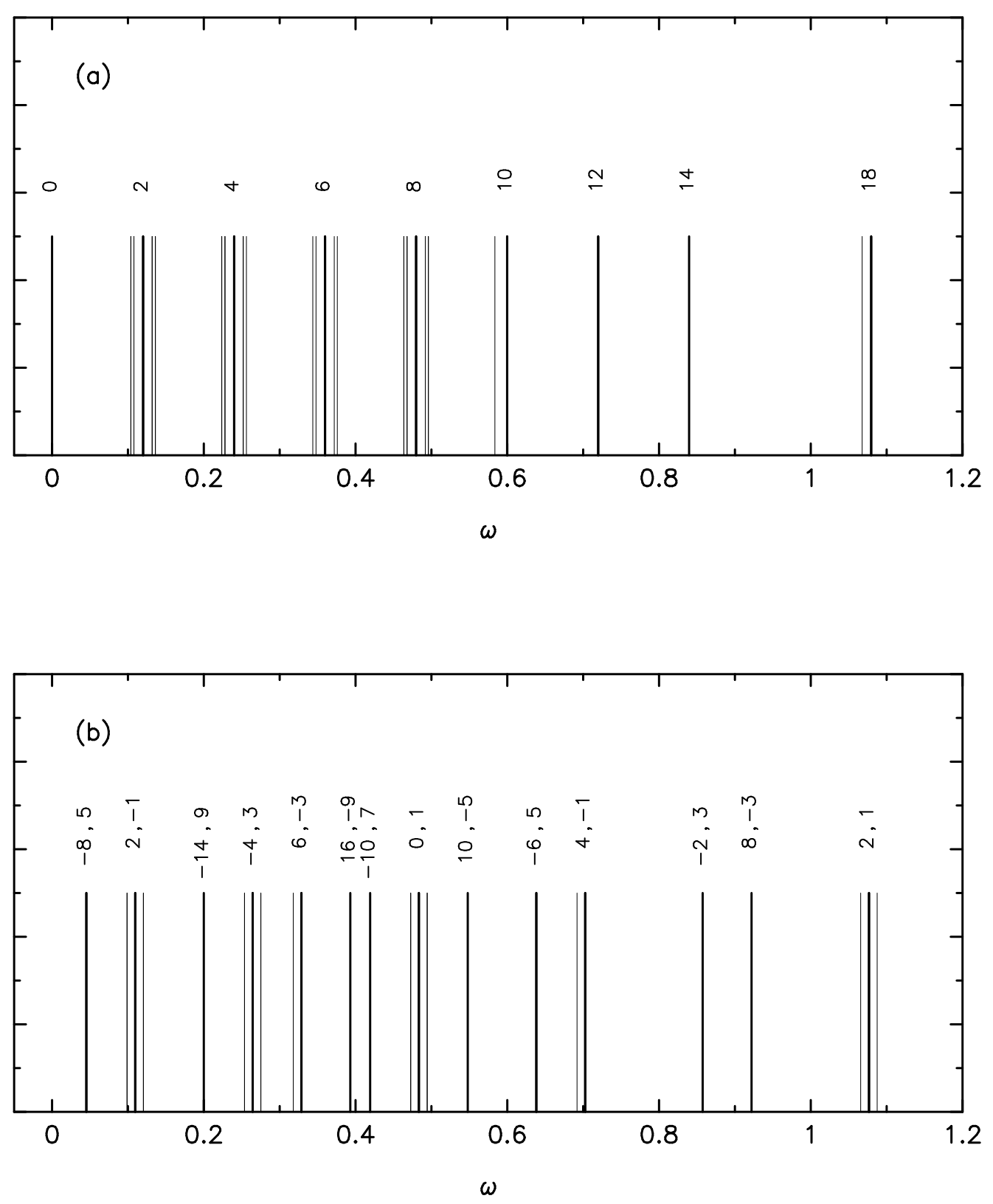

Fig. 6.- 


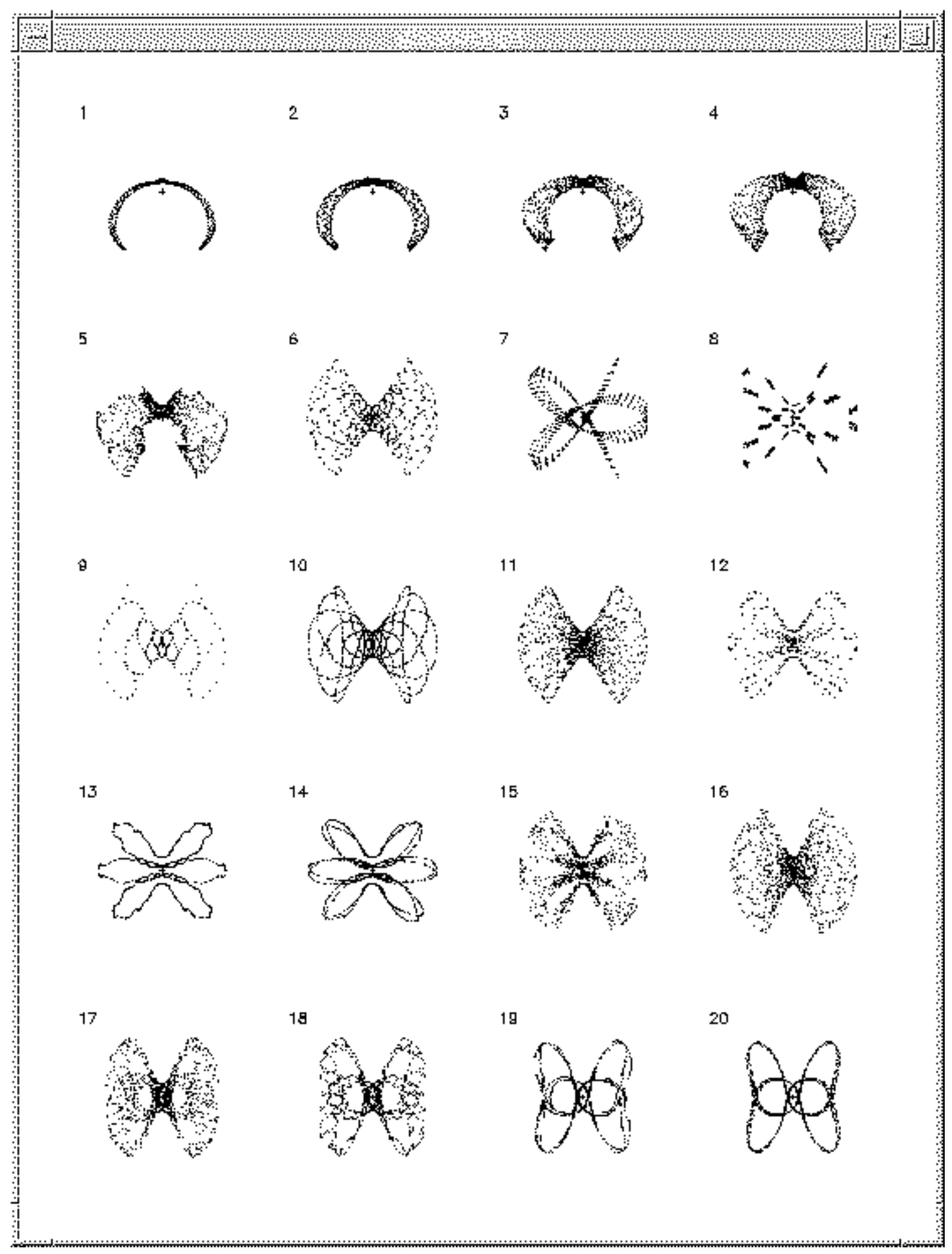

Fig. 7.- 


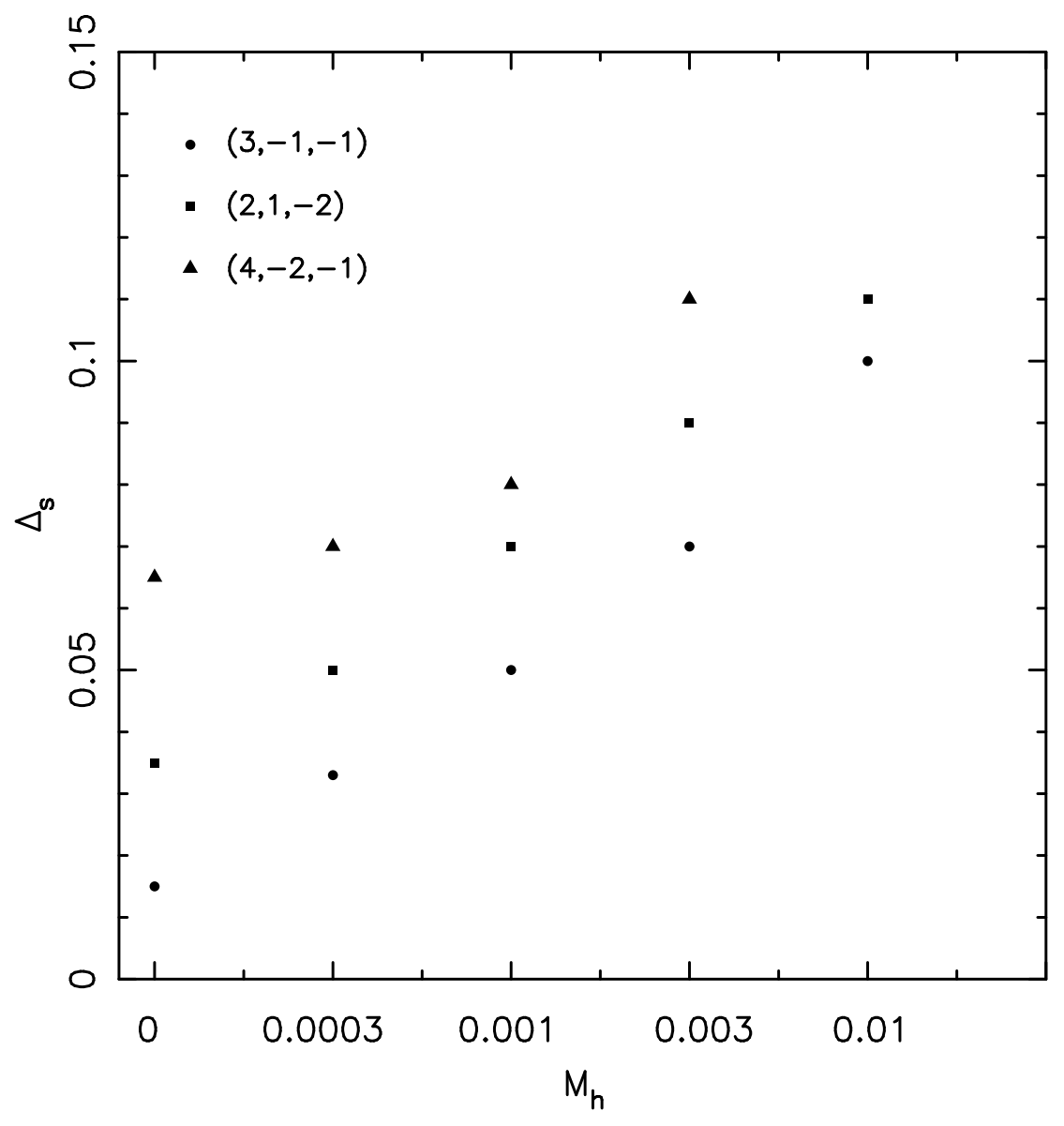

Fig. 8.- 


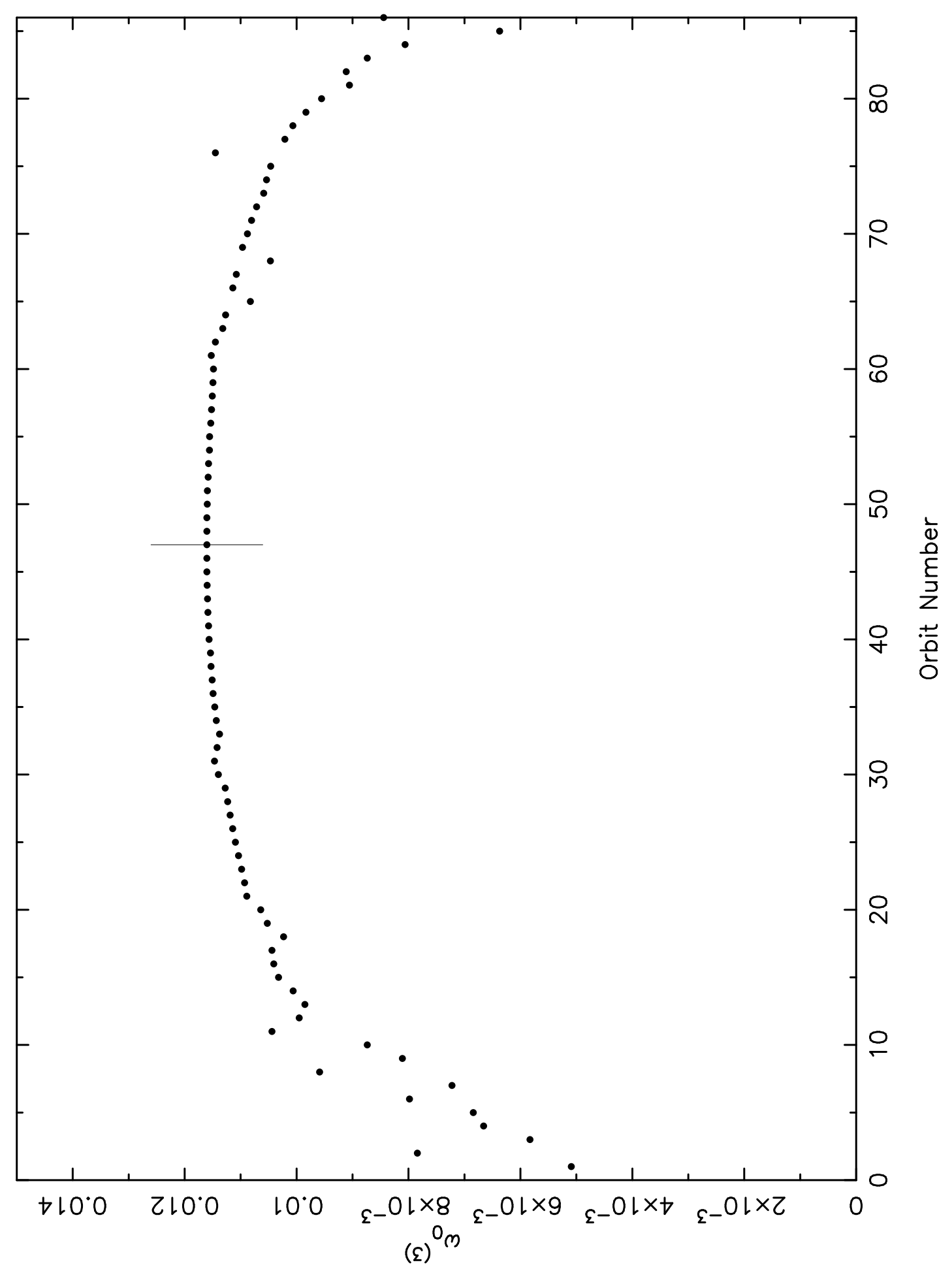

Fig. 9.- 


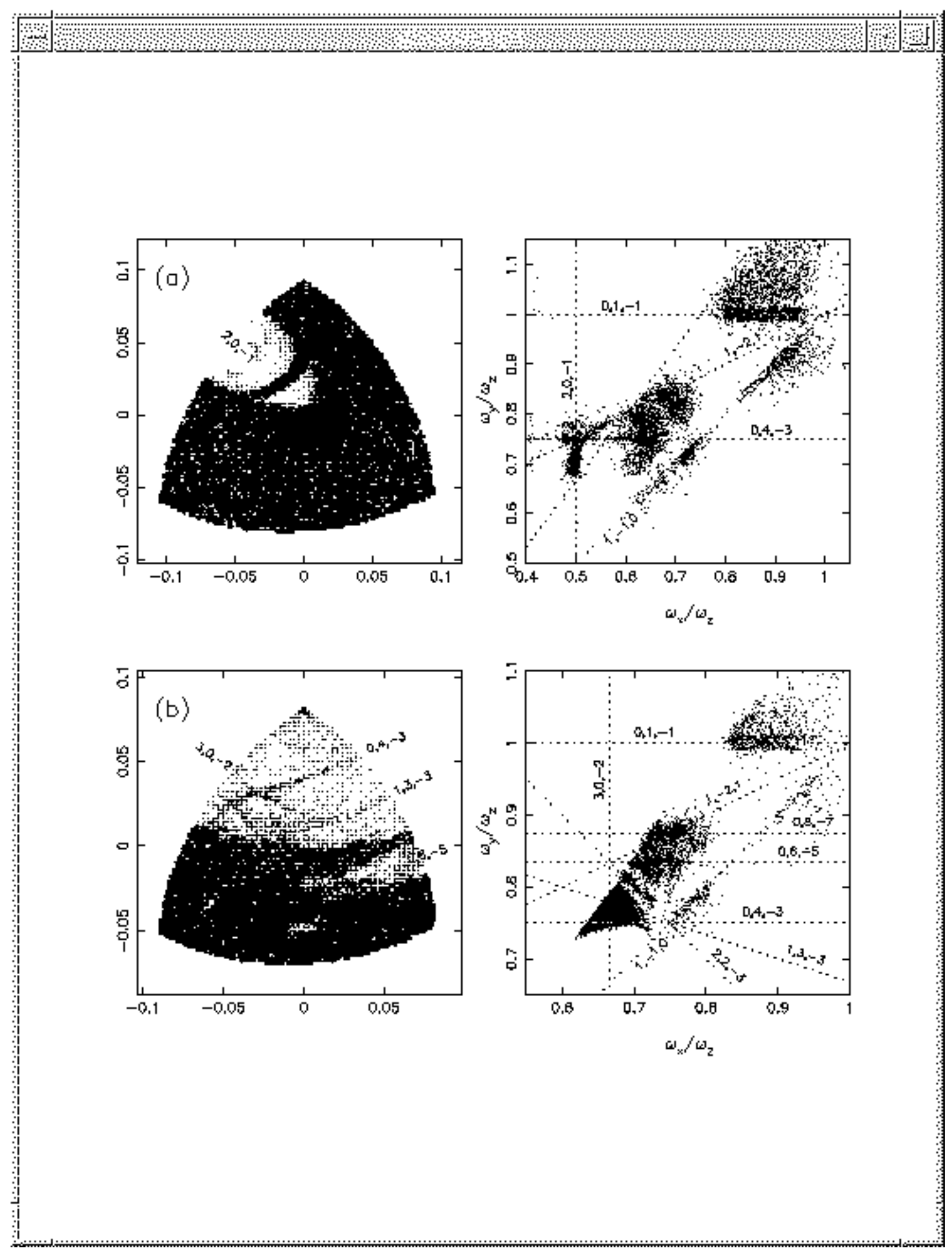

Fig. 10.- 


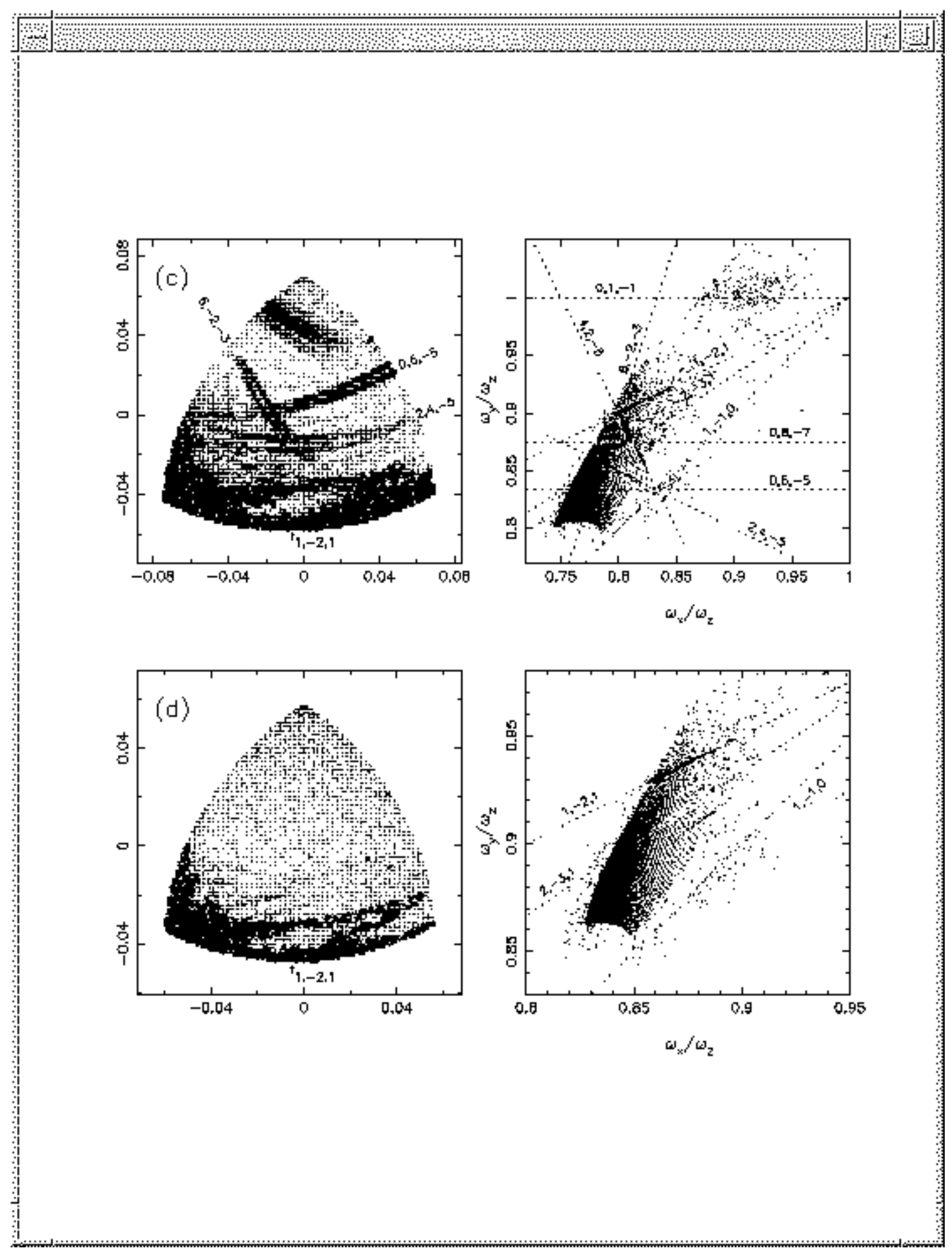

Fig. 10.- 


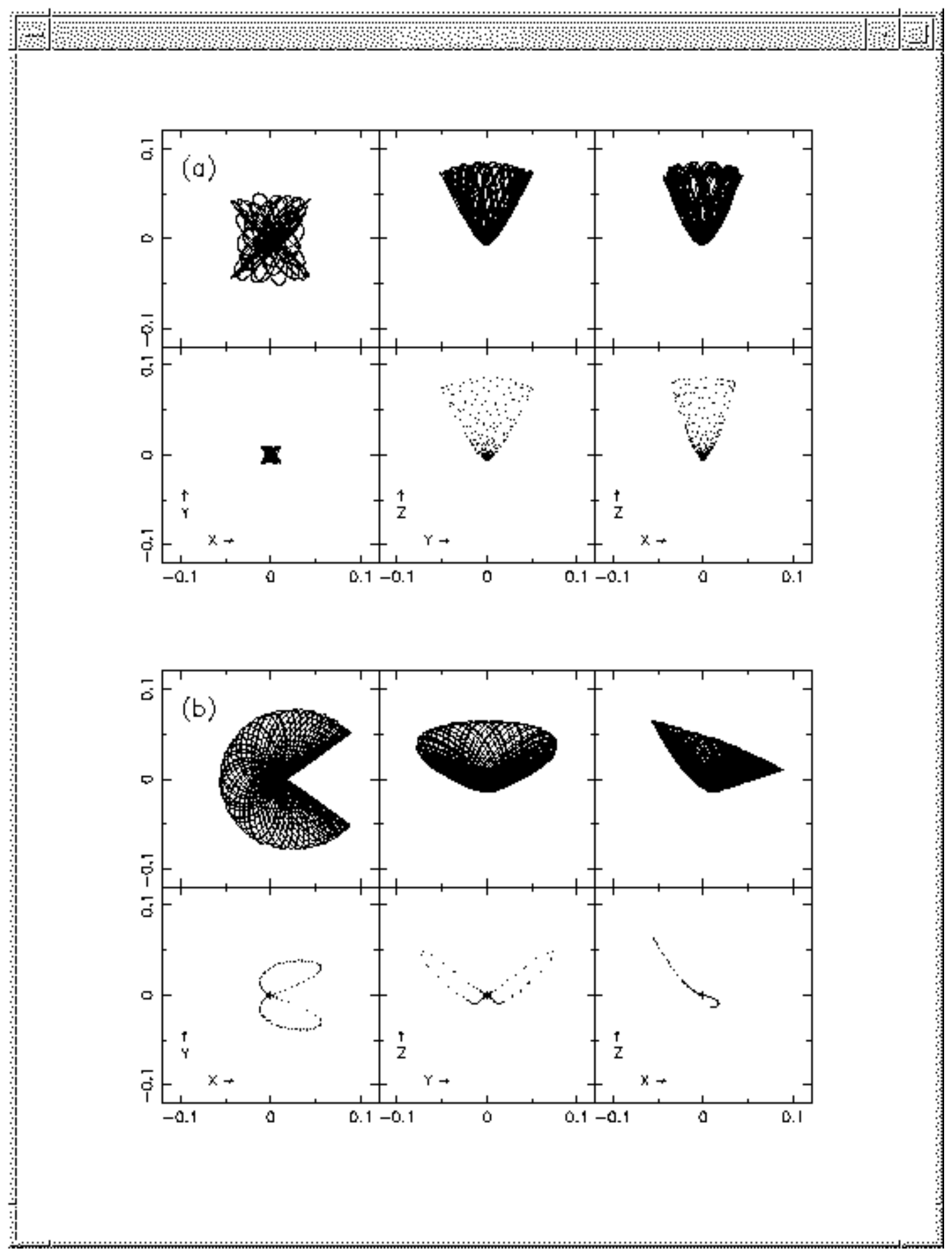

Fig. 11.- 